\title{
LAS CARACTERÍSTICAS NATURALES COMO FACTOR INICIAL PARA UNA CORRECTA PLANIFICACIÓN TERRITORIAL. UNAAPLICACIÓN AL ÁREA FUNCIONAL DE DONOSTIALDEA-BAJO BIDASOA (PAÍS VASCO)
}

\author{
Nagore Dávila Cabanillas \\ nagore.davila@ehu.es
}

\section{RESUMEN}

Dada las características físicas que presenta el Área Funcional de Donostialdea-Bajo Bidasoa y dado el descontrolado consumo de suelo en la misma, se realiza un análisis de los factores naturales más relevantes que influyen en la capacidad de acogida del territorio con el objetivo de proponer nuevas líneas de actuación en los planes de ordenación territorial del área y favorecer un consumo de suelo sostenible. Para ello se aplica el modelo denominado FPEIR como herramienta de análisis territorial.

Palabras clave: características topográficas, valores naturales, red hidrográfica, indicadores, sostenibilidad territorial, modelo FPEIR.

\begin{abstract}
Given the physical characteristics within the functional area of Donostialdea-Bajo Bidasoa and given the uncontrolled consumption of land in it, an anaysis of the most important natural factors influencing the carrying capacity of the territory is carried out with the aim of proposing new courses of action within the spatial plans of the area and promote a sustainable land use. This applies to so-called DPSIR model as a tool for spatial analysis.
\end{abstract}

Fecha de recepción: febrero 2011.

Fecha de aceptación: julio 2012. 
Key words: topography, natural values, river system, indicators, sustainable territorial development, DPSIR model.

\section{INTRODUCCIÓN: INSTRUMENTOS NORMATIVOS EXISTENTES Y ÁMBITO TERRITORIAL DE ESTUDIO}

En lo que respecta a la configuración particular de la Ordenación del Territorio (en adelante OT) en la Comunidad Autónoma del País vasco (CAPV), la Ley 4/1990, de 31 de mayo de Ordenación del Territorio del País Vasco constituye el marco jurídico con la que se desarrolla la política de la OT.

En la exposición de motivos de la propia ley se presentan los objetivos con claridad, entre los que cabe destacar uno específico para el País Vasco: «para paliar los efectos que ha producido el incontrolado crecimiento urbano e industrial soportado por la Comunidad Autónoma del País Vasco fundamentalmente durante la segunda mitad del presente siglo, efectos que han conducido a una importante degradación del nivel de vida de la población ubicada en su territorio» (Exposición de Motivos III. Ley 4/1990, de 31 de mayo de Ordenación del Territorio del País Vasco).

Los instrumentos previstos por la Ley de OT son tres: las Directrices de Ordenación Territorial (DOT), los Planes Territoriales Parciales (PTP) y los Planes Territoriales Sectoriales (PTS). La relación entre las DOT y los PTP's con los PTS's es de jerarquía, de tal modo que ninguna prescripción de este último puede ir en contra de los anteriores (art. 11 LvOT). Asimismo, el PTP tiene en principio el mismo rango que los PTS, pero prevalecen sobre ellos a través de las DOT en su calidad de instrumentos de desarrollo de éstas (art. 17 LvOT).

Las Directrices de Ordenación del Territorio (DOT) se aprobaron mediante Decreto 28/1997, de 11 de febrero, constituyéndose como el marco general de referencia para la formulación de los restantes instrumentos de ordenación territorial y ordenación urbana. La misma Ley de OT señala que su aprobación no es sino la base habilitante para la formulación escalonada de una serie de instrumentos ordenadores que habrán de desarrollarse. Según Ávila Orive (1993, p.67) se trata del «deseo de implicar que el conjunto de objetivos cuya materialización llevan al bienestar de la población no se logra con la promulgación de la ley, sino con la formulación y ejecución que la misma crea».

Sin embargo, a pesar de haberse aprobado en 1997, en 1992 ya se había publicado el documento de Avance (18 de diciembre) y en 1994 la aprobación inicial. Sin embargo, para la aprobación definitiva pasaron 3 años más, en los cuales hubo dos aprobaciones provisionales (1994 y 1996).

Los Planes Territoriales Parciales (PTPs) se redactarán sobre cada una de las Áreas Funcionales (en adelante AAFF) delimitadas por las DOT. Para la delimitación de dichas áreas se han tenido en cuenta además los condicionantes del medio físico que se concretan en unidades con una cierta homogeneidad geográfica, la existencia de unas pautas de comportamiento y actividad de la población, que determinan importantes relaciones de dependencia y complementariedad en el espacio, el sentimiento de pertenencia de sus habitantes y otros factores adicionales que en conjunto han permitido identificar una serie de espacios que se 
han denominado Áreas Funcionales del Territorio, áreas adecuadas para la redacción del planeamiento territorial de escala intermedia (DOT, 1997, p. 301).

No obstante, aunque el procedimiento de iniciación y elaboración es muy similar al establecido para las DOT (avance, aprobación inicial, aprobación provisional y aprobación definitiva) como bien apunta Urkidi (2007), existe un aspecto diferencial en cuanto a quien realiza la iniciativa para la formulación del PTP, es decir, pueden ser desarrollados por el Departamento de Medio Ambiente y Ordenación del Territorio del Gobierno Vasco o por las Diputaciones Forales (en adelante DDFF). Sin embargo, en el caso de que en un Área Funcional (en adelante, AF) queden englobados municipios de provincias diferentes (cabe recordar que las AAFF no son unidades administrativas) la iniciativa corresponde al Gobierno Vasco. Son cuatro las AAFF que integran municipios de otros territorios históricos: Bajo Deba (junto a los municipios guipuzcoanos incluye a los vizcaínos Ermua y Mallabia) Alto Deba (incluye el municipio alavés de Aramaio) Álava Central (municipios vizcaínos de Otxandio y Ubide) y Llodio (integra tantos municipios alaveses, como vizcaínos).

El propio mapa de los PTP's aprobados definitivamente (figura 1) es fiel reflejo de la postura proactiva del Departamento de Medio Ambiente y Ordenación del Territorio del Gobierno Vasco (Urkidi, 2007). Es decir, tal y como se puede observar en la siguiente tabla, de los siete PTP's aprobados cuatro se han desarrollado con una colaboración conjunta entre el Gobierno Vasco y la diputación correspondiente, es decir, por su peculiaridad al abarcar más de un Territorio Histórico, y tal y como preveía la LOTV, han tenido como principal impulsor al propio Departamento de Ordenación del Territorio del Gobierno Vasco, aunque las DDFF también participasen en el mismo (Urkidi, 2007).

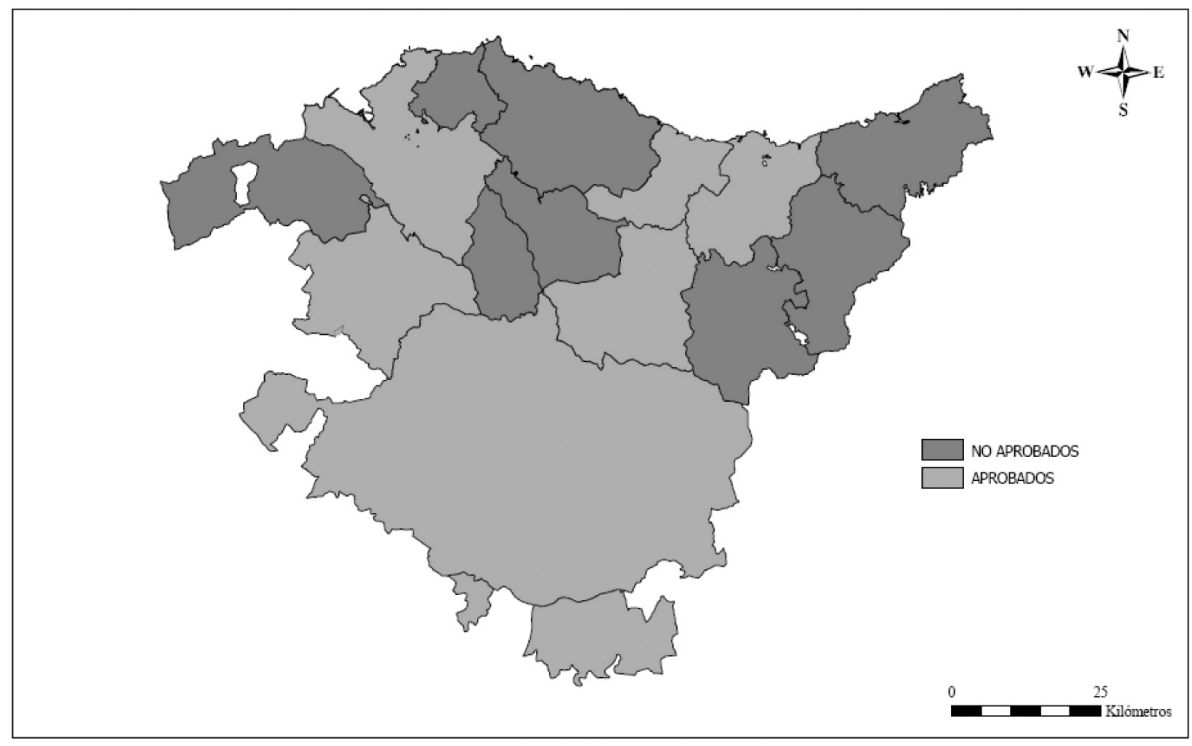

Fuente: Gobierno Vasco. Elaboración propia. 
Bajo la denominación de Plan Territorial Sectorial (PTS) se regulan los distintos instrumentos de ordenación sectorial con incidencia territorial y se puede comprobar la existencia de tres áreas bien definidas: por un lado aquellos que hacen referencia a la ejecución de la política de suelo, por otro aquellos que quedan orientados a las grandes infraestructuras, y por último, los dirigidos a la política medioambiental (Lozano, 2003). Bien sea desde el Gobierno Vasco o desde las diputaciones se han promovido, catorce PTS's, de los cuales ocho ya se han aprobado definitivamente.

El AF que nos ocupa, Donostialdea-Bajo Bidasoa, cuenta con una extensión de $376 \mathrm{~km}^{2}$ y se situá en el extremo nororiental de la CAPV, limitando con Francia. Comprende los términos municipales de Andoain, Astigarraga, Donostia-San Sebastián, Errenteria, Hernani, Hondarribia, Irún, Lasarte-Oria, Lezo, Oiartzun, Pasaia, Urnieta y Usurbil y registra una población de 392.543 habitantes. Debido a su orografía montañosa y por consiguiente escasez de suelo utilizable para muchos usos y actividades, los urbano-industriales han ido localizándose por el corredor de la cadena prelitoral y por todos los valles, formando un continuo urbano que se extiende sin interrupción. Teniendo en cuenta que en estas depresiones y fondos de valle se asienta la mayor parte de los habitantes que registra el AF se intuye la alta concentración que sufren estas. Estas cuestiones y el que, en estos momentos, se esté dando un proceso de revisión del avance al Plan Territorial Parcial de Donostialdea, muestran una gran potencialidad frente a estas líneas de investigación y trabajo geográfico.

\section{OBJETIVOS}

El presente artículo responde a los resultados obtenidos a partir de la realización de la tesis doctoral de la autora del presente trabajo. El objetivo fundamental es realizar un estudio de los elementos naturales del territorio para analizar de qué modo pueden influir o deben influir en la planificación territorial del Área Funcional de Donostialdea-Bajo Bidasoa, así como determinar el riesgo a sufrir algún tipo de amenaza ambiental (riadas, inundaciones...) por parte de los espacios construidos o aquellos que, aun contando con una potencialidad clara, se pueden encontrar insertos en zonas especialmente vulnerables. De este modo, se ofrece al Gobierno Vasco y la Diputación de Gipuzkoa una herramienta de información que pueda servir para la toma de decisiones territoriales, tanto a escalas regionales y comarcales como a locales, a partir de los Planes de Ordenación Urbana de cada uno de los municipios implicados en el Área Funcional.

\section{METODOLOGÍA}

El estudio se ha desarrollado mediante el modelo FPEIR. Responde a las iniciales de Fuerzas Motrices, Presiones, Estado, Impacto y Respuestas y ha sido desarrollado a partir del modelo PER (Presión-Estado-Respuesta), formulado por Friends y Raport en 1979 (Friends, A. y Raport, D. (1979). Es conocido también como DPSIR respondiendo al apócope inglés. Se trata de un método relativamente novedoso, ya que a pesar de haberse utilizado en cuantiosos estudios relacionados con temas medioambientales - «Environmental Indicators» (Organisation for Economic Co-operation and Development, 1994); «Europe's Environment: The Second Assessment», (European Environment Agency, 1998); «Indicators of Sustaina- 
ble Development. Framework and Methodologies», (United Nations, 2001); «Sostenibilidad en España», (Observatorio de Sostenibilidad en España, 2005, 2006 y 2007), «Estado del Medio Ambiente», (Gobierno Vasco, 2004) - , a día de hoy ha sido escasamente utilizado dentro de las etapas de análisis y diagnóstico en referencia a los procesos y planes de ordenación territorial. No obstante, pensamos que ofrece importantes ventajas ya que se basa en la estimación de variables relevantes con un tratamiento muy similar al mostrado por los indicadores territoriales. De hecho, dicha metodología ha sido aplicada con éxito en la Tesis Doctoral recientemente defendida por la autora de la presente comunicación, al alcanzar los objetivos propuestos al inicio de la misma.

Los elementos o variables de análisis se encuentran relacionados como si de una cadena de conexiones se tratase (figura 2), es decir, las fuerzas motrices ejercen presiones sobre el estado del territorio, causando impactos sobre el mismo, la sociedad toma conciencia y responde a estos cambios adoptando políticas de diversa índole, retroalimentando a cada uno de los anteriores elementos y mostrando que la intervención puede ocurrir en cualquier punto del proceso. En muchas ocasiones, en términos formales, se ha descrito como un modelo causal, incluso enfocándose desde el concepto de causalidad. Según Castro (2004) es el enfoque más utilizado para analizar las interrelaciones entre la actividad humana y el equilibrio natural, definiéndolo como una matriz de causa-efecto.

Figura 2

ORGANIZACIÓN DEL MODELO FPEIR (ELABORACIÓN PROPIA)

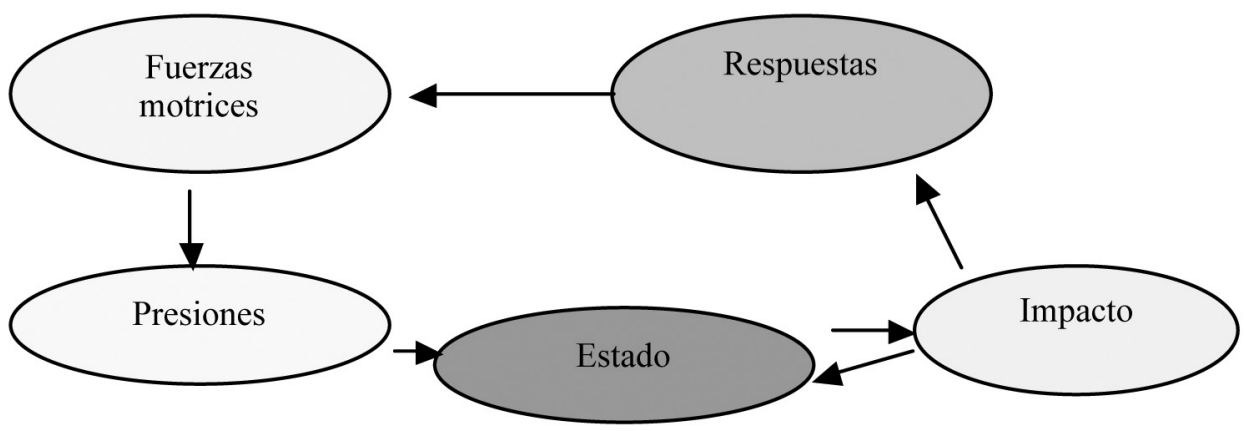

Con este modelo, además, se obtiene una clara imagen respecto al «estado de la situación» (Pino, 2003) mediante los indicadores, y por tanto una perspectiva de análisis y diagnóstico del territorio, por lo que reproduce perfectamente un modelo de relaciones basado en la identificación y caracterización de los problemas y sus causas (United State Environmental Protection Agency, 2006). Es decir, se obtiene una mejor comprensión de estas relaciones, permitiendo a los tomadores de decisiones planificar y gestionar el territorio de manera adecuada, tomando conciencia de las consecuencias potenciales del mismo.

De esta forma, en la aplicación de este modelo, en primer lugar se han identificado las fuerzas motrices que ejercen presión para posteriormente analizar el estado del territorio. Todo ello se ha realizado mediante una batería de indicadores con los cuales se ha podido finalmente observar la tendencia que marca el territorio y que se desarrolla en los siguientes puntos. No obstante y aunque el trabajo original referenciado en la tesis abarca distintas 
variables (más de 15), se ha realizado una discriminación de las que menos interés pudieran mostrar para el objetivo de la presente comunicación, de manera que se abordarán aquellas que mayor preponderancia muestran o las que puedan cumplir los objetos propuestos.

\section{IDENTIFICACIÓN DE LAS CARACTERÍSTICAS NATURALES COMO FUERZA MOTRIZ EN LA DINÁMICA TERRITORIAL DEL ÁREA}

Las características naturales se han considerado como fuerza motriz ya que éstas ejercen unas presiones e impactos de una magnitud significativa sobre el territorio, entre las cuales se debe destacar la limitación de la capacidad de acogida, entendiéndose ésta como «el grado de idoneidad o la cabida del medio para una actividad, teniendo en cuenta, a la vez, la medida en que éste cubre sus requisitos locacionales y los efectos de la actividad sobre el medio. La capacidad de acogida, expresa la relación de la actividad con el medio, en términos de vocacionalidad, compatibilidad o incompatibilidad, por ejemplo» (Gómez Orea, 2002). A continuación se detallan cuales son las fuerzas motrices, presiones e impactos comprendidas dentro de este grupo y los correspondientes indicadores que indican el estado y/o tendencia.

\section{Características topográficas}

El Área Funcional de Donostialdea-Bajo Bidasoa está configurada por valles longitudinales y diversas formaciones montañosas con fuertes pendientes. Estas características físicas del territorio han condicionado la localización de los núcleos de población, siendo los fondos de valle los que presentan un mayor grado de ocupación, registrando de tal modo elevadas cifras de densidad, aspecto que posteriormente se tratará.

\subsection{Indicador de presión: Superficie con pendientes inferiores al $20 \%$}

Este indicador supone una aproximación al grado de presencia de suelo disponible con escasas limitaciones topográficas. El límite se ha establecido en un $20 \%$ ya que a partir de este porcentaje, los costes económicos y las complicaciones en cuanto a accesibilidad y efectos derivados aumentan considerablemente.

\subsection{Evaluación}

Tan solo un 30\% del AF disfruta de unas pendientes inferiores al 20\%. No obstante, si el análisis lo realizamos por municipios, observamos que únicamente Lezo y Lasarte- Oria superan el $50 \%$ de su superficie municipal con pendientes inferiores al $20 \%$.

Asimismo coinciden en que son los únicos que superan el límite establecido (media más la desviación típica de la CAPV) para considerar a un municipio especializado en pendientes inferiores al $20 \%$, calculado en un $49,6 \%$ de la superficie total municipal. 


\subsection{Indicador de estado: Superficie artificializada en pendientes inferiores al $20 \%$}

Para obtener la superficie artificializada en pendientes inferiores y superiores al $20 \%$ de inclinación se han cruzado mediante un SIG la cartografía de pendientes de la CAPV y la cartografía obtenida en Udalplan (capa viviendas, actividades económicas, infraestructuras y equipamiento).

\subsection{Evaluación}

Tal y como queda representado en el mapa inferior (figura 2), un 49,64\% del territorio con una pendiente inferior al $20 \%$ actualmente está artificializado, teniendo en cuenta que las zonas con dicha pendiente representan tan solo el 30\% del total de la superficie del Área Funcional. Se observa por tanto que el suelo susceptible de presentar las características adecuadas para su ocupación, además de resultar un recurso escaso, ya mantiene una alta ocupación. Es decir, el hecho de que la mayor parte de su superficie con pendientes menores del $20 \%$ esté cubierta con áreas residenciales, de actividades económicas y sistemas generales da a entender que futuras ampliaciones urbanas quedan seriamente comprometidas. Esto no significa que no se pueda edificar por encima del nivel de pendientes establecido, pero siempre a costa de costosas obras de acondicionamiento, deterioro ambiental, difícil accesibilidad y riesgos suplementarios en muchos casos. De hecho, en el AF de Donostialdea-Bajo Bidasoa de total de la superficie construida, el $40 \%$ se localiza ya por encima de una pendiente superior al $20 \%$.

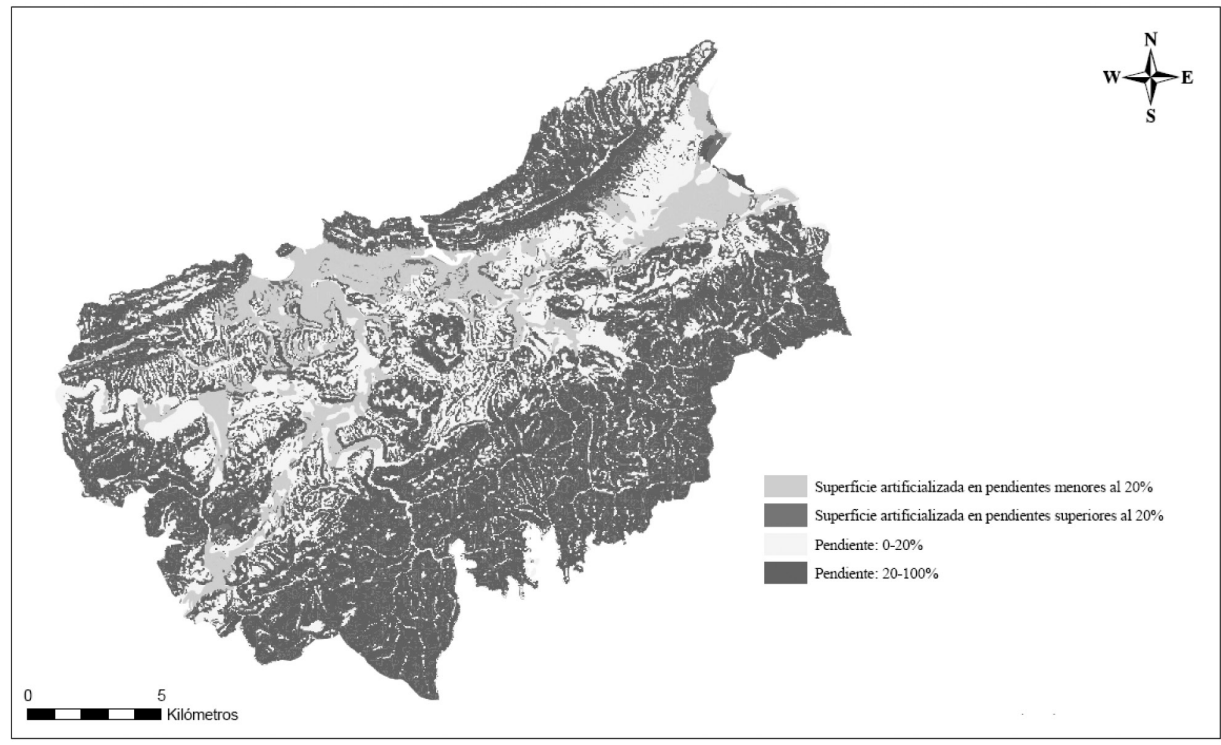

Fuente: Gobierno Vasco y Udaplan. Elaboración propia. 


\section{Valores naturales}

Actualmente, algunos de los valores naturales del AF se encuentran preservados por distintas figuras de protección, tal y como podemos observar en la figura 4. Repasemos pues cuáles son los espacios protegidos y sus planes de gestión.

La vistosa masa granítica de Aiako Harria y sus alrededores, además de ser declarada Parque Natural mediante Decreto 241/95 de 11 de Abril de 1995, con la correspondiente aprobación del Plan de Ordenación de los Recursos Naturales (Decreto 240/95, de 11 de Abril de 1995), ha sido incluido en la lista de Lugares de Interés Comunitario de la Red Natura 2000. Sin embargo, el Plan Rector de Uso y Gestión está actualmente en proceso de elaboración. Por lo tanto, en ausencia de este último, todo tipo de actividad debe ser compatible con las directrices implantadas en el Plan de Ordenación de los Recursos Naturales (en adelante PORN). He aquí los objetivos de mencionado plan.

- La conservación de la Naturaleza y el ordenado aprovechamiento de sus recursos naturales.

- El desarrollo de las actividades agropecuarias y forestales de forma compatible con la conservación y mantenimiento de los recursos naturales.

- El mantenimiento de las actividades agrosilvopescícola como uno de los elementos, responsables del actual estado de conservación ecológica y paisajística de la zona.

- La integración de la calidad local y de los habitantes del área en la gestión del territorio.

- La mejora de la calidad de vida de los habitantes del entorno de Aiako Harria.

- El disfrute colectivo del territorio y el aprovechamiento de sus posibilidades educativas y recreativas, acercando el medio urbano a un medio rural- natural relativamente alejado de su vida cotidiana. En cuanto al uso recreativo, el objetivo primordial de este plan de ordenación está más orientado a la correcta ordenación de la actividad que a su potenciación, promocionando aspectos ligados al recreo y al turismo que pudiesen estas infrautilizados.

Teniendo esto en cuenta, se marcan una serie de normas generales para la regulación de uso y actividades (industrial, agrícola y ganadero, forestal, cinegenéticas y piscícolas, extractivas, recreativas, científicas y de investigación, educativas, constructivas e infraestructuras), así como para la protección de los recursos (suelo, recursos hidrológicos, flora, fauna, paisaje, recursos arqueológicos, antropológicos y arquitectónicos) que deberán ser tenidos en cuenta para cualquier tipo de actividad que quiera desarrollarse dentro de este espacio.

Por otra parte, el río Leitzaran y su valle presentan también unos valores naturales importantes al albergar tanto especies de flora como de fauna poco comunes y al presentar un notable valor geomorfológico debido a su curso meandriforme. Es por ello y por su patrimonio cultural (cromlechs, dólmenes, neveras y ferrerías) por lo que fue declarado Biotopo Protegido mediante el Decreto 416/95, de 29 de Septiembre. Sin embargo, actualmente a falta de un plan de ordenación de los recursos, este biotopo es controlado por las normas que se recogen en el decreto de declaración (BOPV, n 201, de 20 de octubre de 1995). En dicha declaración se concretan cuales son los usos permitidos, los usos autorizables (los usos que el órgano sector del Biotopo podrá autorizar) y los usos prohibidos, tanto para el biotopo como la zona periférica. 
Asimismo, Txingudi, además de declararse ZEPA, está incluido también en la Lista de Humedales de Importancia Internacional, derivada del Convenio de RAMSAR. Desde 1994 tiene aprobado el Plan Especial de Protección y Ordenación del los Recursos Naturales del área de Txingudi (aprobado mediante Orden de 29 de julio de 1994 por el Consejero de Urbanismo, Vivienda y Medio Ambiente del Gobierno Vasco). El plan tiene como objetivos generales:

1. Proteger el paisaje, fauna y flora, manteniendo y potenciando la estructura y dinámica de los ecosistemas.

2. Compatibilizar, en la medida de lo posible, los usos tradicionales.

3. Asumir y minimizar los impactos derivados del uso del suelo ya consolidados, en los casos - cuya eliminación sea desaconsejable económica y socialmente, y proceder a la recuperación de aquellas áreas en las que se estime viable.

4. Facilitar el disfrute público basado en los valores naturales de la zona.

5. Impulsar y programar actividades de investigación y educación ambiental que propicien el conocimiento profundo del patrimonio natural.

Asimismo, existen otros espacios que gozan también de reconocimiento internacional, tal y como lo avala su clasificación como Lugar de Importancia Comunitaria (LIC) y la ya mencionada Zona de Especial Protección para las Aves (ZEPA). Éste es el caso de las terrazas y marismas del Bidasoa, Jaizkibel y el tramo litoral accidentado de Ulia, aunque actualmente todavía no cuentan con sus propias normas de regulación de usos.

Figura 4

ESPACIOS DECLARADOS PROTEGIDOS

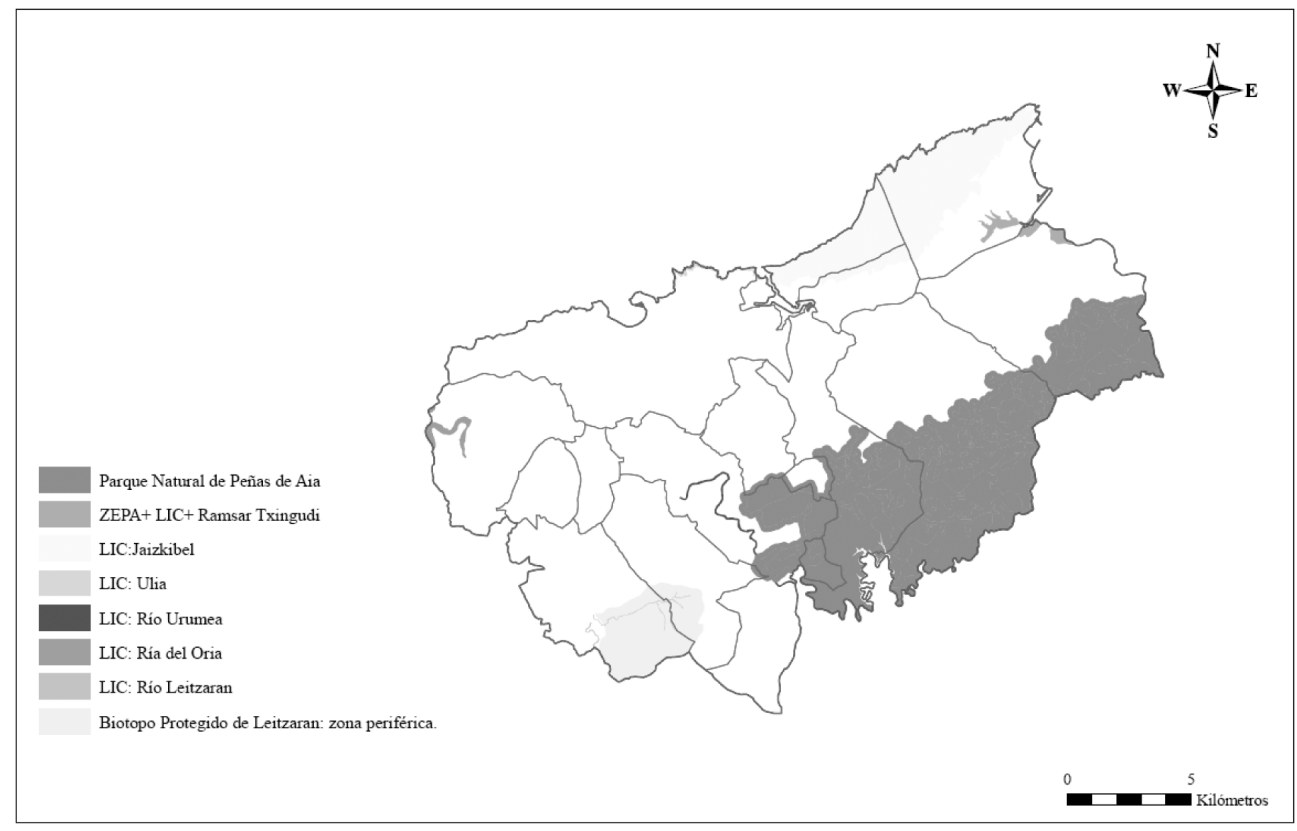

Fuente: Gobierno Vasco. Elaboración propia. 
Del mismo modo, mención especial requiere la inclusión del río Urumea, Oria y Leitzarán en lista de los LIC. La designación viene tratada en el PTS de Ordenación de los márgenes de ríos y arroyos de la CAPV (vertiente cantábrica) al calificarse como «márgenes en zonas de interés naturalístico preferente», determinando una regulación de usos propiciados, permitidos y prohibidos que se recogen en el apartado «D2. Normativa para las márgenes en Zonas de Interés Naturalístico Preferente». En este punto se determina como criterio general de protección el establecimiento de un Área de Protección de Cauce en la que se prohíbe toda operación que implique la alteración del medio.

\subsection{Indicador de presión: Superficie declarada protegida}

Los municipios que destacan (por superar el valor medio del porcentaje de suelo protegido mas media desviación típica: $31,91 \%$ ) en el conjunto de la CAPV, por su porcentaje de superficie municipal catalogada como protegida.

En total, en el AF de Donostialdea-Bajo Bidasoa se registran 98,71 km² declarados como protegidos, es decir, aproximadamente un $26 \%$ de la superficie total del AF. No obstante, tal y como hemos comentado al principio de este apartado, este valor no significa que tal sea la extensión real del espacio protegido, es decir, a pesar de contar con un importante porcentaje de superficie clasificada como protegida, son escasos los planes que se han elaborado para la correcta protección de estos espacios, por lo que ello induce a calcular la superficie que realmente se encuentra protegida.

\subsection{Indicador de estado: Superficie protegida}

A partir del mapa elaborado anteriormente, se calcula la superficie de los espacios que tienen aprobados sus correspondientes planes de ordenación.

\subsection{Evaluación}

Son escasos los planes de gestión u ordenación que están aprobados actualmente en el AF de Donostialdea-Bajo Bidasoa, de hecho, tal y como se puede comprobar en el mapa inferior (figura 5), de ocho espacios declarados protegidos, tan solo dos cuentan con un plan de ordenación: Las Peñas de Aia (Plan de Ordenación de los Recursos Naturales) y Txingudi (Plan especial de protección y ordenación de los recursos naturales).

No obstante, debido a la superficie del Parque Natural de Peñas de Aia $(69,13 \mathrm{~km} 2)$, el $70 \%$ de la superficie declarada protegida ésta regulada bajo un plan de protección y ordenación. Ahora bien, si se calcula respecto al total del Área Funcional el porcentaje disminuye al $18,61 \%$. Si comparamos estos porcentajes con los de la CAPV $(56,34 \%$ respecto a la superficie total protegida y $11,01 \%$ respecto al total del territorio) la valoración para el AF de Donostialdea-Bajo Bidasoa es positiva.

El 7 de diciembre de 2010, se cumplía la fecha límite fijada por la Unión Europea para desarrollar los planes de gestión integrales que garantizaran la conservación de los espacios declarados protegidos y con ellos poder asignar los Lugares de Importancia Comunitaria como Zonas de Especial Conservación. Sin embargo, de las seis Comunidades Autónomas 


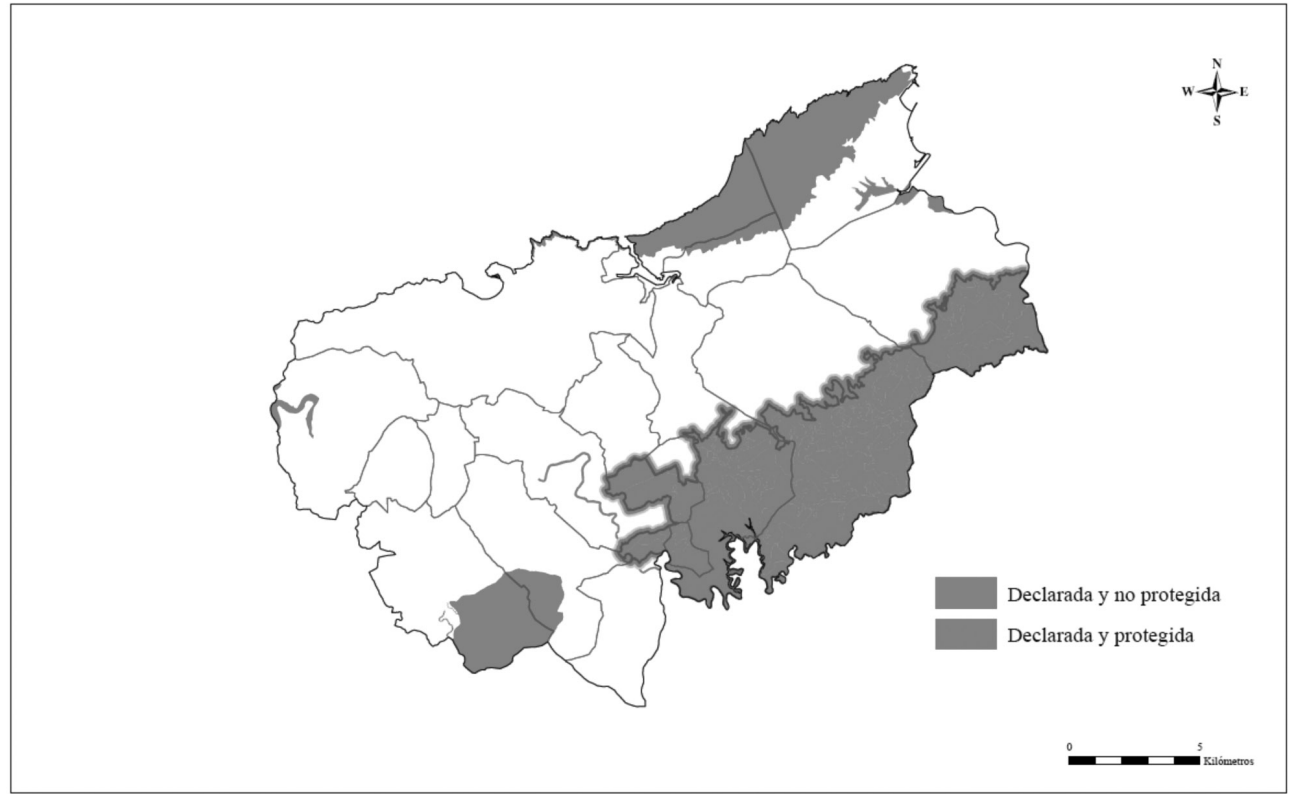

Fuente: Gobierno Vasco. Elaboración propia.

integradas en la región biogeográfica denominada «atlántica» (Asturias, Cantabria, Castilla y León, Galicia, Navarra y País Vasco), tan sólo Navarra ha aprobado algunos de sus planes.

\section{Red hidrográfica}

Teniendo en cuenta las características de la red hidrográfica, ríos de corto recorrido y fuertes pendientes, la presión tratará del riesgo de inundaciones debido a la posibilidad relativamente recurrente de lluvias torrenciales en cuencas donde no han sido respetadas las llanuras de inundación.

Las inundaciones son consideradas como el peligro natural que ha merecido mayor número de actuaciones de ordenación territorial (Olcina, 2004). Evidentemente, para que las inundaciones se conviertan en riesgo deben estar asociadas por un lado a un grado de vulnerabilidad, cuantificada en función del tamaño de la población, y del número y tipo de elementos del medio natural o infraestructuras que pudieran verse afectadas en caso de producirse este tipo de evento (Aguirre, 2005), y por otro lado a un grado de ocurrencia constante.

La ordenación del territorio debe actuar como un instrumento o medida para reducir los riesgos naturales, por lo que la detección y valoración de los posibles riesgos naturales deben constituir un documento básico y necesario para la toma de decisiones sobre la ordenación del territorio. «En la planificación y ordenación del territorio, es de primordial importancia 
que los riesgos existentes sean valorados, y que dicha valoración pueda cartografiarse a una escala apropiada, para conseguir una visión espacial de las zonas más vulnerables y aquellas más seguras» (Aguirre, 2005).

La información extraída de los diferentes periódicos ya revela que muchas de las superficies residenciales e industriales, e incluso infraestructuras de transporte, han sido construidas en zonas inundables. No obstante, para comprobar dicha sujeción analicemos cuáles son las áreas que quedan expuestas a frecuentes episodios de inundación (indicador de presión) y la superficie urbana-industrial que quedaría afectada por éstos (indicador de estado).

\subsection{Indicador de presión: Superficie inundable}

Tal y como se puede observar en el mapa siguiente, la superficie que queda inundada con un periodo de retorno de 10 años es significativa sobre todo en la cuenca del Urumea y Bidasoa. Concretamente en el Urumea, de 487 hectáreas que quedan inundadas con los diferentes periodos de retorno, casi el 70\% (340 ha) de ésta lo hace cada 10 años. En la cuenca del Bidasoa, de 470 ha 329 quedan inundadas con la misma periodicidad, es decir, el 71,50\% de la superficie inundable tiene un periodo de retorno de 10 años. En el resto, concretamente en la cuenca del Oria a su paso por Donostialdea, la superficie inundable cada 10 años no supera el 54\% de la superficie total inundable. Asimismo, en Oiartzun de 122 hectáreas cartografiadas como inundables, 64 tienen un periodo de retorno de 10 años, es decir, un 52\%.

\subsection{Indicador de estado: Superficie residencial e industrial localizada en las llanuras de inundación}

Con la cartografía del PTS de Márgenes de Ríos y Arroyos de la CAPV y Udalplan (2006) se analizan las zonas urbanas que quedan inundadas en periodos de retorno 10, $100 \mathrm{y}$ 500 años, haciendo especial hincapié en las llanuras de inundación de 10 años.

\subsection{Evaluación}

Como se puede observar en el mapa continuo (figura 6), así como en la tabla adjunta al mapa, son varios los tramos urbanizados que quedan afectados por las inundaciones con un periodo de retorno de 10 años, siendo de destacar los tramos de la Michelin en Lasarte-Oria, los sectores de Aguinaga y Zumartegi en Usurbil, en Hernani los polígonos y zonas residenciales de Epele, Lastaola, Ibarluze, Eziago y Orbegozo, en Astigarraga los sectores de Oialuze y Ergobia, los barrios donostiarras de Riberas de Loyola, Txomin Enea y Martutene, así como la zona residencial del noroeste de Irún o la zona de la Fandería de Errenteria.

En este caso, no se ha establecido un umbral de referencia ya que se ha considerado que el hecho de que existan zonas urbanas expuestas a riesgos naturales como son las inundaciones, ya es de por si insostenible. Se ha barajado la posibilidad de obtener el porcentaje de superficie urbana localizada en zonas inundables con periodos de retorno de 10 años para toda la CAPV y poder realizar así una comparación entre el AF de Donostialdea-Bajo Bidasoa y la CAPV, sin embargo, teniendo en cuenta el gran esfuerzo que ha requerido el calcular 


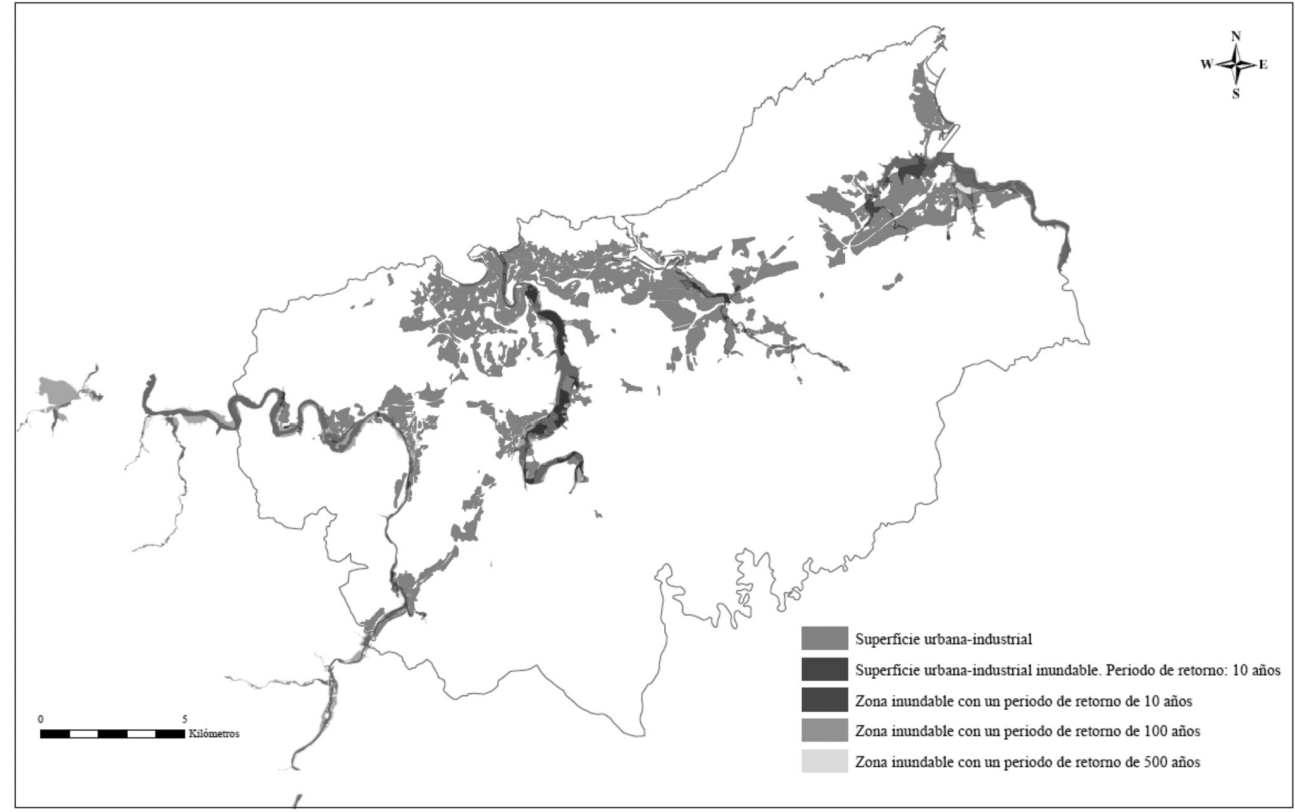

\begin{tabular}{|c|c|c|}
\hline CUENCA & $\begin{array}{c}\text { Zonas inundables ocupados por } \\
\text { usos urbano-industriales }\end{array}$ & $\begin{array}{c}\text { Porcentaje sobre el total de } \\
\text { superficie inundable }\end{array}$ \\
\hline Oria & 6 & $2,50 \%$ \\
\hline Urumea & 112,03 & $33 \%$ \\
\hline Oiartzun & 12,37 & $19 \%$ \\
\hline Bidasoa & 55 & $17 \%$ \\
\hline
\end{tabular}

este porcentaje sobre el AF, el calcularlo sobre toda la comunidad autónoma supondría una muy complicada tarea que supera las pretensiones de este trabajo.

Por todo ello, y considerando desde un punto de vista de la sostenibilidad que ante los riesgos de inundaciones la tolerancia debe ser cero, la valoración es negativa.

\section{SÍNTESIS}

Tal y como se ha podido comprobar, en el AF de Donostialdea-Bajo Bidasoa, debido a su orografía montañosa y por consiguiente escasez de suelo utilizable para muchos usos y actividades, los usos urbano-industriales han ido localizándose por el corredor de la cadena prelitoral y por todos los valles, formando un continuo urbano que se extiende sin interrupción. Teniendo en cuenta que en estas depresiones y fondos de valle se asienta la mayor parte de los 392.543 habitantes que registra el AF se intuye la alta concentración que sufren estas 
zonas y por consiguiente la competencia creciente por parte de los usos residenciales, industriales y de transporte. Son precisamente las áreas más intensamente ocupadas y que mayores densidades de población detectan, las que menor disponibilidad de suelo apto muestran, de manera que municipios como Donostia, Lasarte, Renteria, Pasaia e Irún ocupan los principales puestos negativos, mientras que otros como Usurbil, Urnieta, Oiartzun y Hondarribia se encuentran entre los menos amenazados por esta escasez del recurso. En el medio se situarían el resto de municipios: Hernani, Andoain, Astigarraga y Lezo.

En el 2007, las actividades residenciales e industriales ocupaban aproximadamente el $25 \%$ del total del AF, un porcentaje que desde una perspectiva perceptual puede aumentar. Es decir, tal y como explican Ruiz Urrestarazu y Galdos (2008), desde las partes bajas de los valles los usos predominantes son los residenciales, industriales y de transporte, pero si uno se sitúa, por ejemplo en la cumbre de Peñas de Aia, la sensación de densidad urbanoindustrial se relativiza al contemplar un panorama general con predominio rural en el que los complejos urbanos dibujan cintas alargadas que se acomodan al fondo de los estrechos valles siguiendo las sinuosidades que estos determinan.

La localización de estos usos en los fondos de valle e incluso en las llanuras de inundación, además de suponer una pérdida irreversible de terrenos con una capacidad agrológica muy importante (fluvisoles), ha supuesto catastróficas consecuencias en caso de fuertes inundaciones. Recordemos que los ríos debido a la longitud escasa y a las fuertes pendientes que deben salvar, se caracterizan por su torrencialidad. De hecho, la secuencia temporal entre el momento de precipitaciones máximas y la crecida del caudal es de horas, por consiguiente las crecidas son vertiginosas y violentas.

Por otro lado, los espacios menos atractivos para los usos urbano-industriales son aquellos que presentan un relieve montañoso y de fuertes pendientes. Estas características pueden observarse sobre todo en la unidad del macizo de Cinco Villas, que se caracteriza por ser un terreno abrupto, y en el monoclinal costero de Jaizkibel. En el primero de ellos, predominan los usos forestales: hayedos, robledales y plantaciones forestales, tanto de coníferas como de frondosas. El pastoreo extensivo tiene cierta importancia en las áreas altas y de forma dispersa en el resto. Los caseríos son escasos en el ámbito del espacio, y se distribuyen de forma dispersa. Evidentemente, los usos urbanos e industriales son inexistentes y además están regulados el Plan de Ordenación de los Recursos Naturales del área de Aiako Harria aprobado por Decreto del Gobierno Vasco 240/1995, de 11 de abril. Por otra parte, la cadena montañosa de Jaizkibel (distinguida por la Red Natura 2000 como LIC) queda también al margen de todo desarrollo urbano industrial. El uso principal (en extensión ocupada) del suelo es el pastoreo extensivo (ganado mayor y menor) y en segundo término aparece el uso forestal, propiciando plantaciones de coníferas y en menor medida de frondosas. Asimismo, Ulia, que da continuidad a Jaizkibel, a pesar de su proximidad al núcleo de Donostia-San Sebastián, es destacable por la inexistencia de actividades residenciales e industriales. Efectivamente, se trata de un tramo litoral muy accidentado y con importantes desniveles, por tanto es poco favorable para propiciar desarrollos urbanísticos. 


\section{CONCLUSIONES}

En definitiva, el PTP de Donostialdea debe tener en cuenta la escasa disponibilidad de suelo y reducir las extensiones previstas para procesos de artificialización en Avances anteriores. Y es que, tanto en el Avance del 2003 como en el nuevo documento publicado en el 2008, el modelo territorial que se presenta es más bien un modelo urbanístico que comprende todo el AF, en el que prevalece la ordenación de los usos residenciales, industriales, comerciales y de transporte ante la ordenación inexistente del resto del territorio. El objetivo es conseguir un modelo «descentralizado concentrado», también denominado «ciudad polinucleada en red», un modelo que supone un proceso de difusión totalmente urbanístico, justificando la expansión del perímetro urbano en la necesidad de reequilibrar el empleo y la población (con el objetivo de aminorar la necesidad creciente de desplazamientos motorizados diarios) y en la necesidad de descongestionar las áreas más pobladas y potenciar las menos. Sin embargo esta expansión no parece ser adecuada ante unos índices de artificialización superiores a la media de la CAPV (Dávila, 2010). Es más, en los documentos se puede observar la poca pretensión por parte del PTP de cortar con la dinámica expansiva urbanística que en el AF de Donostialdea-Bajo Bidasoa se ha ido registrando durante las dos últimas décadas.

\section{BIBLIOGRAFÍA}

AGENCIA EUROPEA DE MEDIO AMBIENTE (1998): Europe's Environment: The Second Assessment. AEMA.

AGUIRRE MURUA, G. (2005): «La valoración de los riesgos en la ordenación del territorio: Metodología práctica». Boletín de la Asociación de Geógrafos Españoles, $\mathrm{n}^{\circ} 40$, 393-405.

ALLENDE LANDA, J. (2006): «La ordenación del territorio en la CAPV. Directrices de Ordenación Territorial. Análisis crítico y nuevas propuestas». Informes-diagnósticos DOT. Gobierno Vasco.

CASTRO BOLOÑA, J. (2004): Indicadores de desarrollo sostenible urbano. Una aplicación para Andalucía. Universidad de Málaga.

DAVILA CABANILLAS, N. (2010): La planificación territorial como herramienta sostenible del territorio. Los Planes Territoriales Parciales de Donostialdea-Bajo Bidasoa y Gernika-Markina. Universidad del País Vasco-Euskal Herriko Unibertsitatea. Tesis Doctoral. Documento inédito.

FRIENDS, A. y RAPPORT. D. (1979): «Towards a comprehensive framework for environmental statistics: a stress-response Approach». Statistics Canada Catalogue, 11-510.

GOBIERNO VASCO (2004): Estado del Medio Ambiente. Vitoria-Gasteiz. Servicio de Publicaciones del Gobierno Vasco/Departamento de Medio Ambiente y Ordenación del Territorio.

GÓMEZ OREA, D. (2002): Ordenación Territorial. Mundi Prensa. Madrid.

LOZANO VALENCIA, P. (2003): «La ordenación territorial en el País Vasco: debilidades, amenazas, fortalezas y oportunidades de la escala intermedia». Investigaciones Geográficas, $\mathrm{n}^{\mathrm{o}} 32,157-176$. 
OBSERVATORIO DE SOSTENIBILIDAD EN ESPAÑA (2005): Sostenibilidad en España. Madrid. Mundi-Prensa Libros.

OBSERVATORIO DE SOSTENIBILIDAD EN ESPAÑA (2006): Sostenibilidad en España. Madrid. Mundi-Prensa Libros.

OBSERVATORIO DE SOSTENIBILIDAD EN ESPAÑA (2007): Sostenibilidad en España. Madrid. Mundi-Prensa Libros.

OLCINA CANTOS, J. (2004): «Riesgo de inundaciones y ordenación del territorio en la escala local. El papel del planeamiento urbano municipal». Boletín de la Asociación de Geógrafos Españoles, n⿳3 37, 49-84.

ORGANIZACIÓN PARA LA COOPERACIÓN Y DESARROLLO ECONÓMICO (1994): Environmental Indicators: OECD Core Set. Paris. OCDE.

PINO NECULQUEO, M (2002): Análisis de indicadores de sostenibilidad ambiental y urbana en la Agenda 21 Local y ecoauditorias municipales. El caso de las regiones urbanas europeas. Universitat Politécnica de Catalunya.

RUIZ URRESTARAZU, E. y GALDOS URRUTIA, R. (2008): Geografía del País Vasco. San Sebastián. Nerea.

UNITED STATES ENVIRONMENTAL PROTECTION AGENCY (2006): Strategy for Indicator Development. Border 2012: U.S. Mexico.Environmental Program Report. United State Environmental Protection Agency, Secretario de Medio Ambiente y Recursos Naturales.

URKIDI ELORRIETA, P. (2007): Análisis del tratamiento del medio físico en el Planeamiento Territorial de la CAPV (1940-2006). Universidad del País Vasco. 